\title{
Factores de riesgo asociados a las complicaciones del tratamiento quirúrgico de los quistes aracnoideos intracraneales en pacientes adultos. Análisis retrospectivo de
}

\author{
una serie de casos
}

\author{
A. Vega; F. Cohn; J. Diegopérez y E. Zepeda
}

Servicio de Neurocirugía. Hospital de Especialidades. Centro Médico Nacional Siglo XXI. IMSS. México.

\section{Resumen}

Introducción. Analizamos los resultados quirúrgicos en pacientes adultos operados de quiste aracnoideo intracraneal, evaluando las diferentes técnicas quirúrgicas empleadas, la vida media de cada procedimiento y el índice de complicaciones postquirúrgicas. Este estudio describe los resultados de nuestra institución.

Material y métodos. Incluimos a todos los pacientes operados con diagnóstico de quiste aracnoideo intracraneal desde enero de 2001 hasta diciembre del 2006. Se evaluó la eficacia de cada técnica quirúrgica empleada y la vida media de cada procedimiento, así como los eventos adversos asociados al tratamiento quirúrgico.

Resultados. La distribución por género fue 15 hombres y 13 mujeres, con un promedio de edad de 34 años y con un rango de 17 a 65 años al momento de la cirugía. El síntoma predominante fue la cefalea. La localización de los QA fue: 24 supratentoriales y 4 infratentoriales. Se realizaron 48 cirugías; se presentaron complicaciones postquirúrgicas en 11 pacientes del grupo de estudio. La craneotomía fue el procedimiento con una vida más prolongada (1245 días). La vida media de la craneotomía más fenestración fue significativamente mayor que el resto de los procedimientos (853 vs 193 días con una P: $\mathbf{0 . 0 3 3 3 )}$ y el quiste aracnoideo grado III de Galassi es un factor de riesgo para reoperación en nuestro grupo de estudio con una razón de momios de 13.417.

Conclusión. La fenestración por craneotomía fue en la experiencia de este hospital el procedimiento con menores complicaciones y mayor tiempo de efectividad. Las complicaciones del tratamiento quirúrgico están fuertemente relacionadas con la ubicación y el tamaño del quiste.

PALABRAS CLAVE: Quiste aracnoideo intracraneal. Tratamiento quirúrgico. Resultados quirúrgicos.
Risk factors associated with the complications of surgical treatment of intracranial arachnoid cysts in adult patients. Retrospective case series analysis.

Summary

Introduction. We analyzed the surgical results in patients operated for an arachnoid cyst evaluating the different surgical techniques employed, the average life of each procedure and the rate of postoperative complications. This study describes the results from our institution.

Material and methods. We included all patients operated with a diagnosis of intracranial arachnoid cyst from January 2001 to December 2006. We evaluated the effectiveness of each surgical technique and the average life of each procedure, as well as adverse events associated with surgical treatment.

Results. The gender distribution was 15 men and 13 women, with an average of 34 years and with a range of 17 to 65 years at time of surgery. The predominant symptom was headache. The location of the arachnoid cyst was 24 supratentorial and 4 infratentorial. 48 surgeries were performed. There were complications in 11 patients in the study group; the craniotomy was the procedure with a longer life (1245 days). The half-life of the craniotomy more fenestration was significantly higher than the rest of the procedures (853 vs. 193 days with a P: 0.0333) and the arachnoid cyst grade III of Galassi is a risk factor for reoperative in our study group with an odds ratio of 13,417.

Conclusion. In our experience the fenestration by craniotomy was the procedure with fewer complications and increased time of effectiveness. Treatment complications are strongly related to cyst location and size.

KEY WORDS: Intracranial arachnoid cyst. Surgical treatment. Surgical results.

Abreviaturas. QA: quistes aracnoideos 


\section{Introducción}

Los quistes aracnoideos (QA) son colecciones benignas de líquido cefalorraquídeo ${ }^{24,29,32}$. La mayoría se detectan en las primeras dos décadas de la vida y se diagnostican con menos frecuencia en pacientes adultos y ancianos $^{11,12}$. Pueden aparecer a lo largo del neuroeje en las distintas áreas anatómicas donde existe aracnoides ${ }^{35}$. Las manifestaciones clínicas dependen de diversos factores como son la edad del paciente, la ubicación y el tamaño del quiste.

El objetivo fundamental del tratamiento se encamina al control de la sintomatología, que desaparece en la mayoría de los casos aunque no se consiga reducir de forma significativa el tamaño del quiste ${ }^{17,28}$. Existe una gran controversia en cuanto a la técnica quirúrgica que se debe emplear: la cirugía abierta mediante craneotomía con fenestración de las membranas ${ }^{2,4,10,17,22}$, la derivación del contenido del quiste a otra cavidad ${ }^{6,14,16,20}$ y la fenestración del quiste asistida por endoscopía ${ }^{5,8,21,25,33}$.

Se estudiaron los resultados quirúrgicos en un grupo de pacientes adultos con diagnóstico de quiste aracnoideo intracraneal analizando las diferentes técnicas quirúrgicas empleadas, la vida media de cada procedimiento y el índice de complicaciones postquirúrgicas con la finalidad de conocer los principales factores de riesgo asociados al tratamiento quirúrgico.

\section{Material y métodos}

Este estudio retrospectivo analiza un grupo de 28 pacientes adultos con diagnóstico de quiste aracnoideo intracraneal, los cuales fueron tratados quirúrgicamente en el Servicio de Neurocirugía del Hospital de Especialidades Centro Médico Nacional Siglo XXI de la Ciudad de México en el periodo comprendido de Enero de 2001 hasta Diciembre del 2006, con un seguimiento promedio de 28 meses.

Los resultados se obtuvieron analizando las diferentes técnicas quirúrgicas empleadas; la eficacia del tratamiento se valoró en base a si el primer procedimiento quirúrgico fue suficiente para resolver la sintomatología inicial del paciente. La vida media de cada procedimiento se definió como el intervalo de tiempo en días entre dos procedimientos quirúrgicos o la última nota de evolución asentada en el expediente clínico y las complicaciones se definieron como los eventos adversos debidos a un procedimiento quirúrgico.

La ausencia de desaparición del QA (o de los síntomas que se le atribuyeron) se catalogó como complicación del tratamiento quirúrgico cuando el paciente fue sometido a una reintervención quirúrgica para resolución de esta situación.

\section{Resultados}

El universo de estudio estuvo constituido por un total de 28 pacientes, distribuidos por género en 15 hombres y 13 mujeres, con un promedio de edad de 34 años (rango de 17 a 65 años) y con una mediana de 30.5 años.

La localización de los QA con respecto a la tienda del cerebelo fue: 24 supratentoriales (16 en fosa media, 6 de la convexidad, 1 supraselar y 1 intraventricular) y 4 infratentoriales ( 2 en hemisferio cerebeloso, 1 en el ángulo pontocerebeloso y 1 en la lámina cuadrigémina). A su vez los QA de la fosa media los agrupamos de acuerdo a la clasificación de Galassi: Galassi I: 2, Galassi II: 4 y Galassi III: 10 .

El síntoma predominante fue la cefalea en 15 pacientes, seguido de crisis convulsivas en 6 pacientes, síntomas visuales y déficit motor en 2 pacientes, 1 paciente con espasmo hemifacial y 2 pacientes asintomáticos con QA de gran tamaño.

No hubo un sólo patrón para definir la conducta quirúrgica por lo que al revisar los datos encontramos que se llevaron a cabo un total de 48 cirugías: fenestración por craneotomía (22), fenestración por craneotomía más derivación cisto-peritoneal (1), craneotomía más fenestración endoscópica (2), derivación cisto peritoneal (14), fenestración endoscópica más derivación cisto peritoneal (1), punción guiada por estereotaxia (1), derivación ventrículo-peritoneal (1), fenestración endoscópica (1), ventriculostomía (2), drenaje de hematoma por trépanos (2) y lobectomía temporal (1).

Diecisiete pacientes presentaron resolución del QA con una sola intervención, mientras que los 11 pacientes restantes precisaron más de un procedimiento quirúrgico.

Las complicaciones posteriores a la cirugía se presentaron en 11 pacientes del grupo de estudio incluidos aquellos pacientes con fallo primario del tratamiento quirúrgico (5), disfunción valvular (8), hematoma subdural (3), hematoma epidural (1), infección de herida quirúrgica (1), edema cerebral (1), infarto de la arteria cerebral media (1), hidrocefalia (2) y muerte (2). (Tabla 1)

Cuando se analizó la efectividad del tratamiento se encontró una duración promedio en días antes de una nueva cirugía de 292 días, independientemente del tipo de procedimiento quirúrgico. Sin embargo, cuando se realiza el análisis de sobrevida por tipo de procedimiento, se encontró que la fenestración por craneotomía fue el procedimiento con una vida media más prolongada (1245 días) seguido por la derivación cisto-peritoneal más endoscopia (502 días), fenestración por craneotomía más derivación cisto-peritoneal (402 días), derivación ventrículoperitoneal (98 días), derivación cisto-peritoneal (97 días), otros procedimientos (63 días) (Figura 1).

La duración más prolongada de los procedimientos qui- 
Tabla1

Complicaciones postquirúrgicas

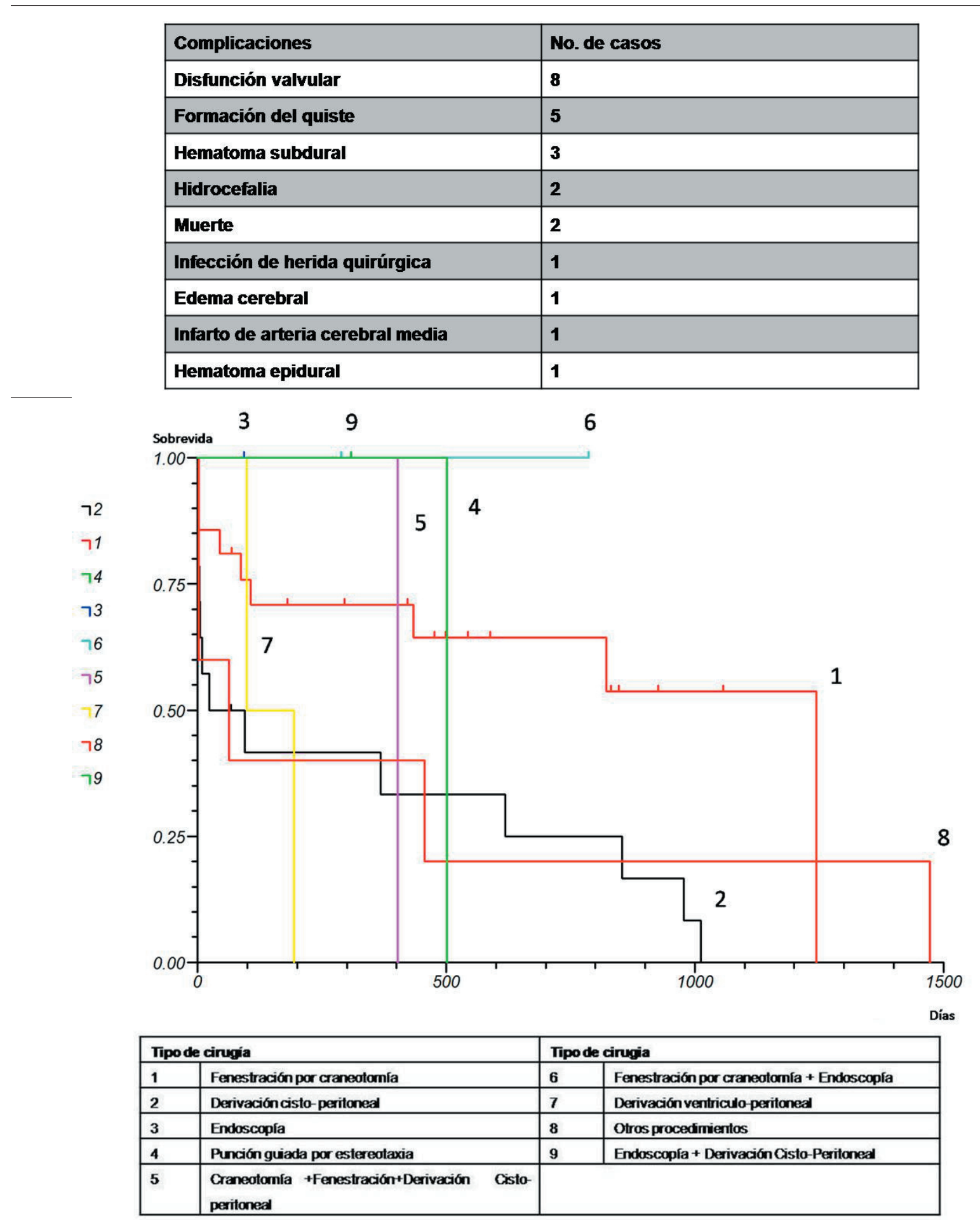

Figura 1. Sobrevida del total de procedimientos quirúrgicos.

rúrgicos fue estadísticamente diferente cuando se comparó la fenestración por craneotomía con la derivación cisto- peritoneal y esta diferencia fue la única estadísticamente significativa (razón de momios de 2.84082 con un intervalo 


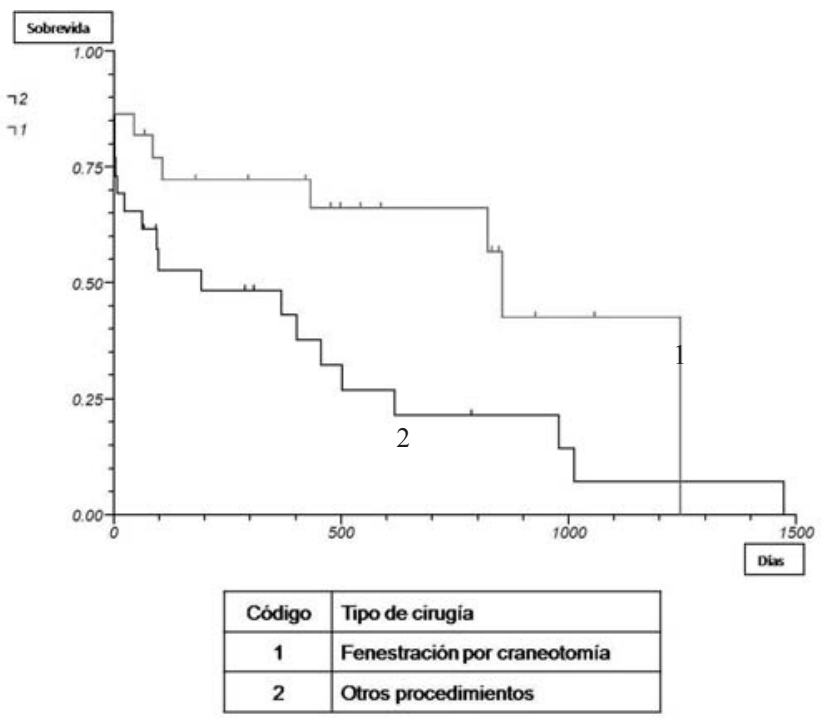

Figura 2. Comparación de sobrevida "Craneotomía vs Otros"

de confianza del $95 \%$ de 1.175957 a 6.862716 ). Cuando se comparó cualquiera de los otros procedimientos entre sí no existió diferencia significativa. Hay que señalar que muchos de los grupos no tuvieron número suficiente para realizar una comparación válida.

Cuando analizamos la curva de sobrevida de la fenestración por craneotomía comparándola con el resto de los procedimientos quirúrgicos encontramos que la fenestración por craneotomía tiene una vida media de 853 días mientras que el resto de procedimientos quirúrgicos tienen una vida media de 194 días. Siendo la diferencia estadísticamente significativa y con un valor de $\mathrm{P}=0.0333$ (Figura 2).

Además evaluado desde la perspectiva del riesgo, es dos veces más frecuente una reoperación cuando no se utilizó la fenestración por craneotomía (Riesgo relativo $=2.168153$ con IC 95\% de 1.072236 a 4.384192). Nótese que en la gráfica hay puntos más claros de diferencia a partir del primer año después del procedimiento y esta diferencia tiende a ser mayor conforme pasa el tiempo (Figura 3).

Al realizar el análisis del riesgo de una nueva cirugía respecto al tamaño y localización del QA, se evaluaron diferentes posibles combinaciones pero sólo cuando había un quiste Galassi III vs cualquier otra localización o tamaño; encontramos que hay un riesgo de 13.417 (razón de momios con IC: del 95\%: 3.041 a 59.193). Esto quiere decir que es 13 veces más probable que el paciente se reopere cuando tiene un QA Galassi III, independientemente de la técnica quirúrgica empleada.

\section{Discusión}

Los QA tienen una incidencia del 1\% de las lesiones

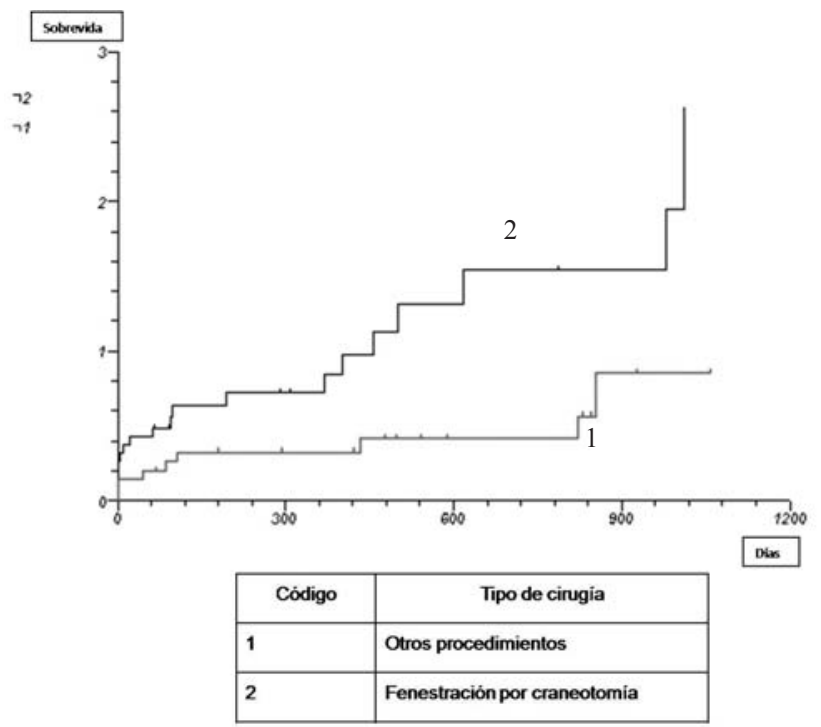

Figura 3. Riesgo de falla "Craneotomía Vs otro procedimiento"

intracraneales. Son más frecuentes en varones que en mujeres y la mayoría se detecta en las dos primeras décadas de la vida ${ }^{32}$. En nuestra serie la distribución por sexo y grupo de edad siguen ese mismo patrón. La localización más frecuente es en el hemisferio izquierdo y con predilección por la fosa media (30-50\%). En nuestros resultados observamos una incidencia más alta en cuanto a la localización de los QA de la fosa media de hasta un 66\%.

La localización y el tamaño del QA están frecuentemente asociados al cuadro clínico; sin embargo, hasta el momento no hay evidencia que relacione a los QA con la sintomatología que presentan los pacientes al momento del diagnóstico, ya que una buena parte de los QA son detectados en forma incidental. En nuestra serie el síntoma principal fue la cefalea, sin embargo, no hay una forma inequívoca de relacionar a la cefalea con la presencia de un quiste aracnoideo, por lo que no se podría asegurar que el paciente mejore con el procedimiento quirúrgico si la presencia de este síntoma es el que origina la decisión quirúrgica. Debido a esto, debemos considerar una actitud expectante en los quistes de pequeño volumen y asintomáticos que se diagnosticaron al practicar un estudio neurorradiológico por otro motivo ${ }^{27}$, sin embargo, se ha documentado alteración en el metabolismo y perfusión del tejido cerebral circundante al quiste, en pacientes clínicamente silentes con normalización de estos parámetros posterior a la descompresión del quiste ${ }^{7,26}$.

En lo que respecta al manejo de los QA, los puntos fundamentales son: la indicación de tratamiento (quirúrgico vs vigilancia) y la elección de la técnica quirúrgica más adecuada. Dentro de las opciones de tratamiento quirúrgico se encuentran la punción-aspiración del quiste, 
la fenestración del quiste y la comunicación de éste con el espacio subaracnoideo ya sea mediante craneotomía o asistido por endoscopia, y por último la derivación del liquido desde el quiste hacia otras cavidades donde el líquido pueda reabsorberse. Independientemente de la técnica quirúrgica empleada el objetivo principal consiste en la descompresión del quiste con o sin derivación asociada del contenido del QA. La elección de cualquiera de estos procedimientos quirúrgicos depende de la experiencia y preferencia del cirujano.

El tratamiento quirúrgico está indicado en los QA sintomáticos, bien por producir hipertensión intracraneal, hidrocefalia o síntomas focales, así como en aquellos quistes que presentan un crecimiento progresivo en las pruebas de imagen y en el caso de debutar con hemorragia intraquística ${ }^{1,11,23,24}$.

La fenestración del quiste a través de cualquier técnica (craneotomía o endoscopia) a menudo es preferida antes que colocar un sistema de derivación para evitar las complicaciones relacionadas con éste ${ }^{30}$. La fenestración por craneotomía permite la inspección y escisión de la pared del quiste, la coagulación de vasos sanguíneos, fenestración de quistes septados, confirmación histopatológica con un procedimiento de toma de biopsia y en determinados lugares la fenestración del quiste hacia las cisternas basales ${ }^{4,17}$.

La escisión completa de las membranas del quiste es el tratamiento lógico si tenemos en cuenta las propiedades secretoras de la pared del quiste ${ }^{15}$ pero por desgracia, rara vez se puede llevar a cabo debido a la estrecha relación anatómica entre la membrana y el tejido cerebral subyacente, por lo tanto, algunos autores sugieren una apertura selectiva con resección limitada de la membrana del quiste. Este procedimiento es eficaz para limitar el flujo de líquido cefalorraquídeo al espacio subdural o subaracnoideo ${ }^{30}$.

La fenestración por craneotomía está indicada, sobre todo, en los QA de fosa media por la facilidad de establecer una comunicación entre la cavidad quística y las cisternas basales y es obligada su realización si el quiste debuta con un hematoma intraquístico o subdural ${ }^{23}$. La fenestración micro quirúrgica mediante craneotomía es un método seguro y eficaz para el tratamiento de los QA de fosa media. Este procedimiento se puede realizar con un mínimo de morbilidad a través de una minicraneotomía con instrumentos micro quirúrgicos estándar ${ }^{22}$.

Por otra parte, los quistes aracnoideos puede ser tratados con éxito colocando una derivación cisto-peritoneal que permite el vaciamiento progresivo del quiste hacia el peritoneo, favorece una reexpansión progresiva del cerebro circundante y reduce las posibles complicaciones derivadas de la descompresión brusca del cerebro, esto principalmente con el uso específico de válvulas de presión programable ${ }^{3,14}$. Es un método seguro pero se debe de reco- nocer los riesgos de obstrucción, infección y dependencia del sistema valvular ${ }^{24}$.

La derivación cisto-subdural es un procedimiento mínimamente invasivo para el tratamiento de QA supratentoriales en pacientes adultos con buenos resultados a largo plazo ${ }^{16,34}$.

Diversos autores informan de que ambos procedimientos; fenestración y derivación, son igualmente eficaces para el tratamiento de los quistes aracnoideos intracraneales y hacen referencia a que los factores que realmente influyen en los resultados son la reducción del volumen y la ubicación del quiste más que la modalidad terapéutica ${ }^{19}$. En nuestro estudio el principal procedimiento quirúrgico utilizado fue la fenestración por craneotomía en un $52.9 \%$ seguido de la derivación cisto- peritoneal en un 31.3\%.

El tratamiento de los quistes aracnoideos se ha revolucionado con la introducción de técnicas endoscópicas. Actualmente la neuroendoscopia se ubica como un procedimiento mínimamente invasivo y ha demostrando su eficacia y seguridad en el tratamiento de los QA, por lo que diversos autores recomiendan la fenestración endoscópica como procedimiento de primera elección para el trata-

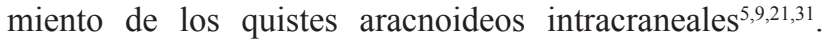
En nuestra serie, los pacientes que fueron sometidos a tratamiento endoscópico ya sea solo o como método auxiliar de otro procedimiento quirúrgico, no ameritaron una segunda intervención quirúrgica, sin embargo, por ser un número reducido de procedimientos no alcanzan a ser estadísticamente significativos.

Howard et $\mathrm{al}^{18}$ analizan los resultados quirúrgicos de 650 pacientes con diagnóstico de QA en 21 series reportadas en la literatura de 1980 a 1997.394 pacientes fueron sometidos a una fenestración por craneotomía, mientras que 188 pacientes fueron sometidos a un tipo de derivación cisto-peritoneal; 30 pacientes, la mayoría asociados con hidrocefalia secundaria fueron sometidos a una derivación ventrículo-peritoneal o ventrículo-atrial. La combinación de fenestración por craneotomía más una derivación cistoperitoneal fue utilizada como procedimiento primario en otros 30 pacientes y en los 8 pacientes restantes otros procedimientos no especificados.

Los índices de reoperación fueron reportados de la siguiente manera: $19.9 \%$ para la fenestración por craneotomía, $28.6 \%$ para la derivación cisto-peritoneal, $75 \%$ para la derivación ventrículo-peritoneal o ventrículoatrial, $42.9 \%$ para la fenestración por craneotomía más derivación cisto-peritoneal y $9.1 \%$ para otros procedimientos. En lo que respecta a nuestros pacientes el índice de reoperación fue $80 \%$ para la derivación cisto peritoneal y $35 \%$ para la fenestración por craneotomía como procedimientos principales realizados en nuestra serie.

Al igual que en otras series de pacientes adultos con QA intracraneales las complicaciones del tratamiento qui- 
rúrgico están relacionadas con la ubicación y el tamaño del quiste, ya que éstas se producen casi exclusivamente en pacientes portadores de QA Galassi tipo III ${ }^{4,16,17}$. De un total de 16 pacientes con QA de fosa media, 13 fueron intervenidos mediante fenestración por craneotomía, de éstos, 4 precisaron una segunda cirugía, los 4 con QA Galassi tipo III. Los 3 pacientes restantes (con QA tipo III de Galassi) que se trataron de primera instancia con una derivación cisto-peritoneal necesitaron una segunda intervención quirúrgica.

Las complicaciones reportadas por Howard et al ${ }^{18}$ asociadas al tratamiento con fenestración por craneotomía fueron del $10.2 \%$, mientras que para la derivación cistoperitoneal fue de $16.9 \%$ con una mortalidad del $0.8 \%$. En nuestra serie la morbilidad fue de un $39.2 \%$ y la mortalidad de $7.1 \%$.

\section{Conclusiones}

La fenestración por craneotomía fue el procedimiento quirúrgico que se realizó con mayor frecuencia. No es de sorprender dado que en la experiencia de nuestro Hospital, es el procedimiento con menos complicaciones y mayor tiempo de efectividad.

Las complicaciones del tratamiento quirúrgico en nuestros pacientes están directamente relacionadas con la ubicación y el tamaño del quiste, ya que éstas se produjeron casi exclusivamente en pacientes portadores de QA Galassi tipo III respecto a cualquier otra localización.

\section{Bibliografía}

1. Arai, H., Sato, K., Wachi, A., Okuda, O., Takeda, N.: Arachnoid cysts of the midle cranial fossa: experience with 77 patients who were treated with cystoperitoneal shunting. Neurosurgery. 1996; 39: 1108-1112.

2. Artico, M., Cervoni, L., Salvati, M., Fiorenza, F., Caruso, R.: Supratentorial arachnoid cysts: clinical and therapeutic remarks on 46 cases. Acta Neurochir (Wien) 1995; 132: 75-78.

3. Belliard, H., Roux, F.X., Turak, B., Nataf, F., Deavaux, B., Cioloca, C.: The Codman Medos programmable shunt valve. Evaluation of 53 insertions in 50 patients. Neurochirurgie 1996; 42:139-146.

4. Boutarbouch, M., El Ouahabi, A., Rifi, L., Arkha, Y., Derraz, S., El Khamlichi, A.: Management of intracranial arachnoid cysts: institutional experience with intal 32 cases and review of the literature. Clin Neurol Neurosurg 2008; 110: $1-7$.

5. Choi, J.U., Kim, D.S., Huh, R.: Endoscopic approach to arachnoid cyst. Childs Nerv Syst. 1999; 15: 285-291.

6. Ciricillo, S.F., Gogen, P.H., Harsh, G.R., Edwards, M.S.: Intracranial arachnoid cysts in children. A comparison of the effects of fenestration and shunting. J Neurosurg 1991; 74: 230-235.

7. De Volder, A.G., Michel, C., Thauvoy, C., et al.: Brain glucose utilisation in acquired childhood aphasia associated with a sylvian arachnoid cyst: recovery after shunting as demonstrated by PET. J Neurol Neurosurg Psychiatry 1994; 57: 296-300.

8. Di Rocco, F., Yoshino, M., Oi, S.: Neuroendoscopic transventricular ventriculostomia in treatment for intracranial cyst. J Neurosurg 2005; 103: 54-60.

9. Gangemi, M., Colella, G., Magro, F., Maiuri, F.: Suprasellar arachnoid cysts: endoscopy versus microsurgical cyst excision and shunting. Br J Neurosurg 2007; 21: 276-280.

10. Galassi, E., Tognetti, F., Franko, F.: Infratentorial arachnoid cyst. J Neurosurg 1985; 63: 210-217.

11. Gelabert-González, M.: Quistes aracnoideos intracraneales. Rev Neurol 2004; 39: 1161-1166.

12. Gelabert, M., García, A., et al.: Quiste aracnoideo interhemisférico en el anciano. Neurocirugía 1997; 8: 233236.

13. Gelabert, M., Prieto, A., González-García, J., Abdulkader, L., Pravos, A.G., García, A.: Quiste aracnoideo intraventricular en el adulto. Rev Neurol 1997; 25: 10951098.

14. Germano, A., Caruso, G., Caffo, M., et al.: The treatment of large supratentorial arachnoid cysts in infants with cystperitoneal shunting and Hakim programmable valve. Childs Nerv System 2003; 19: 166-173.

15. Go, K.G., Houthoff, H.J., Blaauw, E.H., Having, P., Harsuiker, J.: Arachnoid cyst of the sylvian fissure. Evidence of fluid secretion. J Neurosurg 1984; 60: 803-810.

16. Helland, C.A., Wester, K.: Arachnoid cysts in adults: long-term follow-up of patients treated with internal shunts to the subdural compartment Surg Neurol 2006; 66: 56-61.

17. Helland, C.A., Wester, K.: A population based study of intracranial arachnoid cyst: clinical and neuroimaging outcomes following surgical cyst decompression in adults. J Neurol Neurosurg Psychiatry 2007; 78: 1129-1135.

18. Howard, H.K.: Complications related to the surgical treatment of arachnoid cyst. En: Kaufman HH (ed): Cerebrospinal Fluid Collections. Park Ridge, IL: AANS, 1998, pp 139147.

19. Kandenwein, J.A., Richter, H.P., Borm, W.: Surgical therapy of symptomatic arachnoid cysts-an outcome analysis. Acta Neurochir (Wien) 2004; 146: 1317-1322.

20. Kim, S.K., Cho, B.K., Chung, Y.N., Kim, H.S., Wang, K.C.: Shunt dependency in shunted arachnoid cyst: a reason to avoid shunting. Pediatr Neurosurg 2002; 37: 178-185.

21. Kirollos, R.W., Javadpour, M., May, P., Malluci, C.: Endoscopic treatment of suprasellar and third ventricle-related arachnoid cysts. Childs Nerv Syst 2001; 17: 713-718.

22. Levy, M.L., Wang, M., Aryan, H.E., Yoo, K., Meltzer, H.: Microsurgical keyhole approach for middle fossa arach- 
noid cyst fenestration. Neurosurgery 2003; 53: 1138-1145.

23. Mori, K., Yamamoto, T., Horinaka, N., Maeda, M.: Arachnoid cyst is a risk factor for chronic subdural hematoma in juveniles: twelve cases of chronic subdural hematoma associated with arachnoid cyst. J Neurotrauma. 2002; 19: 1017-1027.

24. Oberbauer, R.W., Haase, J., Pucher, R.: Arachnoid cyst in children: a European co-operative study. Childs Nerv Syst 1992; 8: 281-286.

25. Schroeder, H.W., Gaab, M., Niendorf, W.R.: Neuroendoscopic approach to arachnoid cysts. J Neurosurg 1996; 85 : 293-298.

26. Sgouros, S., Chapman, S.: Congenital middle fossa arachnoid cysts may cause global brain ischaemia: a study with 99Tc- hexamethylpropyleneamineoxime single photon emission computerised tomography scans. Pediatr Neurosurg 2001; 35: 188-194.

27. Sommer, I.E.C., Smit, L.M.: Congenital supratentorial arachnoidal and giant cysts in children: a clinical study with arguments for a conservative approach. Childs Nerv Syst 1997; 13: 8-12.

28. Soukup, V.M., Patterson, J., Trier, T.T., et al.: Cognitive improvement despite minimal arachnoid cyst decompression. Brain Dev 1998; 20: 589-593.

29. Sundaran, C., Paul, T.R., Raju, B.V., Ramakrishna, M.T., Sinha, A.K., Prasad, V.S., Purohit, A.K.: Cyst of the Central Nervous System: a clinic pathologic study of 145 cases. Neurol India 2001, 49: 237-242.
30. Tamburrini, G., Caldarelli, M., Massimi, L., Santini, P., Di Rocco, C.: Subdural hygroma: an unwanted result of Sylvian arachnoid cyst marsupialization. Childs Nerv Syst 2003; 19: $159-165$.

31. Tirakotai, W., Schulte, D.M., Bauer, B.L., Bertalanffy, H., Hellwig, D.: Neuroendoscopic surgery of intracranial cysts in adults. Childs Nerv Syst. 2004; 20: 842-851.

32. Villarejo, F.: Quistes aracnoideos intracraneales. En: Villarejo F, Martínez-Lage J, eds. Neurocirugía Pediátrica. Madrid, Ergon, 2001, pp. 127-130.

33. Wang, J.C., Herer, L., Soumeidane, M.: Advance in the endoscopic management of suprasellar arachnoid cyst in children. J Neurosurg 2004; 100: 418-426.

34. Wester, K.: Arachnoid cysts in adults: experience with internal shunts to the subdural compartment. Surg Neurol 1996; 45: 15-23.

35. Wester, K.: Peculiarities of intracranial arachnoid cysts: Location, sidedness, and sex distribution in 126 consecutive patients. Neurosurgery 1999; 45: 775-779.

Vega A.; Cohn, F.; Diegopérez J.; Zepeda E.: Factores de riesgo asociados a las complicaciones del tratamiento quirúrgico de los quistes aracnoideos intracraneales en pacientes adultos. Análisis retrospectivo de una serie de casos. Neurocirugía 2009; 20: 454-460

Correspondencia postal: Dr. Alfonso Vega Sosa. Rosselló 446, $6^{\circ}$ $4^{\circ}$. CP 08025. Barcelona España. 